Canadian Food Studies

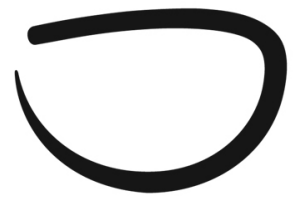

La Revue canadienne des études sur l'alimentation

Original Research Article

\title{
First foods as Indigenous food sovereignty: Country foods and breastfeeding practices in a Manitoban First Nations community
}

\author{
Jaime Cidro ${ }^{a *}$, Tabitha Robin Martens ${ }^{b}$, Lynelle Zahayko ${ }^{b}$, Herenia P. Lawrence ${ }^{c}$ \\ a University of Winnipeg, Department of Anthropology \\ ${ }^{b}$ University of Manitoba, Faculty of Social Work \\ c University of Toronto, Faculty of Dentistry
}

\begin{abstract}
As a concept and in practice, Indigenous food sovereignty (IFS) offers insights into the social, cultural, and environmental challenges of a deficient food system. The associated poor health outcomes of this system include infant and child health issues such as early childhood caries and childhood obesity, and are a grave concern in many First Nations communities. Extant research has failed to consider the role of infant feeding traditions as an element of Indigenous food sovereignty. Breastfeeding and country food (also called traditional food or cultural food) consumption among infants has been long practiced in First Nations communities, resulting in healthier infants. The research described in this article originated with a research project called the Baby Teeth Talk study (BTT). This is a community-based trial which is testing a prenatal/post-natal behavioural and preventive intervention for early childhood caries (ECC) among pregnant First Nations women and their infants in urban and on-reserve communities in Ontario and Manitoba. In Norway House Cree Nation, located in northern Manitoba, research participants shared stories on the methods used by caregivers for oral health care, including breastfeeding promotion. This paper reviews the literature relating to IFS, breastfeeding, and the introduction of country food to infants. Through the voices of grandmothers in one community in northern Manitoba, Canada, we connect the introduction of country food and breastfeeding to the larger IFS movement and positive health outcomes for infants, and improve the conceptualization and practice of IFS.
\end{abstract}

Keywords: breastfeeding, First Nations, infant feeding, Indigenous food sovereignty, country food, infant oral health 


\section{Introduction}

Indigenous Food Sovereignty (IFS) is a term that has only recently become conceptualized in research, but has long been practiced in First Nations communities (Morrison, 2011). Food sovereignty, or the increased control over food systems, has special resonance for First Nations people whose rights continue to be eroded despite the existence of treaties. As a concept and in practice, IFS offers insight into the challenges of a deficient food system that includes high prices for fruits and vegetables and other items in local stores, changes in the availability of traditional foods due to access issues, landscape changes, and concerns about the health of the soil, plants, and wildlife, to name a few (Skinner, 2006; Willows, 2005).

Numerous studies have highlighted the disparity between First Nations communities and non-First Nations communities with regards to food security. First Nations households, both on reserve and off, face higher levels of food insecurity than non-First Nations households. While food security considers issues around access and availability of food, it has been criticized for failing to appropriately capture the importance of traditional foods. Where food security ensures people have enough food to eat, Indigenous food sovereignty argues for active participation in Indigenous food systems so that the mind, body, and spirit are nourished (Martens, 2015).

Food issues are certainly linked to the alarming rates of poor health outcomes plaguing First Nations people in Canada. Type 2 diabetes (Reeds et al., 2016), cardiovascular disease (Tobe, Maar, Roy, \& Warburton, 2015) and hypertension (Reading, 2015) are all examples of health issues that have emerged in recent decades that can be traced to larger social determinants of health, including food security and more broadly IFS. Infant and child health issues such as early childhood caries and childhood obesity are a grave concern in many First Nations communities. In First Nations communities, these health issues can be placed in a larger social, political, economic and cultural context of food sovereignty.

The extant research has not, to date, considered the role of infant feeding traditions as an element of Indigenous food sovereignty. Breastfeeding and "country food” (also called traditional food or cultural food) consumption among infants has been long practiced in First Nations communities resulting in healthy infants and children who grew to be healthy adults. Recent research in Canada has looked at food programs in First Nations communities as examples of IFS. For instance, the Urban Aboriginal Garden Project in British Columbia has been documented (Mundel \& Chapman, 2010), while country foods initiatives, like those in Tukisigiarvik in Nunavut and northern Manitoba have been examined as well (Lardeau, Healey, \& Ford, 2011; Thompson, Kamal, Alam, \& Wiebe, 2012). However, these studies focused on adult participation, and there is no mention of the importance of early food sovereignty, in utero, for infants and young children.

As with all components of First Nations food systems, the processes of colonization including the reserve land structure, residential schools, and the Sixties Scoop have all interrupted these traditional infant feeding practices such as breastfeeding and the early introduction of country food. This has impacted the ability of First Nations communities not only 
to practice IFS as being connected to their larger cultural practices, but identify the loss of Indigenous infant feeding practices as an integral part of what Chandler and Dunlop refer to as “cultural wounds” which impacts identity formation (2014, p. 78).

The basis for this research comes from discussion around culturally based interventions for early childhood caries (ECC) which emerged during a research project being led by Dr. Herenia P. Lawrence at the University of Toronto, called the Baby Teeth Talk study (BTT). The BTT is an on-going community-based ECC trial which is testing a multi-component pre-natal and post-natal behavioural and preventive intervention among pregnant First Nations women and their infants living in urban and on-reserve communities in Ontario and Manitoba (Lawrence et al., 2004; 2008; 2016). In Norway House Cree Nation, a community located in northern Manitoba, research participants began sharing stories with the community researchers on the methods used by caregivers in terms of oral health care, including breastfeeding promotion. As a result, an additional research project was formulated to look at these cultural approaches to infant feeding and oral health practices, with a focus on integrating these approaches into community programs for the current population of parents. A wide range of themes related to infant feeding emerged. An unanticipated finding was the issue of IFS and first foods for infants.

Cultural continuity is embedded in IFS principles and practice and are especially poignant when they are operationalized beginning with a new life. Breastfeeding and early introduction of country foods not only reinforces cultural continuity but also addresses issues of food insecurity which many Indigenous communities face, especially in rural, remote and northern communities. This paper highlights the importance of resituating IFS by looking at breastfeeding and the introduction of country foods through a brief review of literature. By incorporating the voices of grandmothers in one community in northern Manitoba, Canada, we can make the connection between the introduction of country food and breastfeeding to the larger IFS movement and positive health outcomes for infants and young children and resituate the conceptualization and practice of IFS.

\section{Indigenous food sovereignty}

As a written theory Indigenous food sovereignty is based on the concept of food sovereignty. Food sovereignty was first defined in a visible way by La Via Campesina, an international movement of small-farm workers, peasant, and Indigenous peoples as a response to power struggles in the global food system ${ }^{1}$. The protection and redistribution of land are key to food sovereignty, along with the recognition that Indigenous people should have the right to be on the land to produce food (Martens, 2015). Food sovereignty aims to link production with consumption, and argues for the sustainable use of land through local and traditional knowledge.

\footnotetext{
${ }^{1}$ See, for example, Desmarais’s 2007 book Globalization and the Power of Peasants: La Vía Campesina. Halifax, NS: Fernwood Publishing.
} 
These concepts are not new to First Nations communities; however, over time, First Nations food systems and relationships with the land have been altered through the impacts of colonization and other traumatic experiences such as residential schools and the Sixties Scoop (Martens, 2015). Indeed, the loss of knowledge around traditional food harvests and resource management practices is seen as an impact of the "exposure of First People to colonialism and imperial pressures” (Turner \& Turner, 2008). The introduction of processed foods along with landscape changes such as climate change; contamination and hydro development have further altered these food systems, and help to understand the motivations of the IFS movement. Morrison (2008) defines an Indigenous food system as "land, soil, water, air and culturally important plant, fungi and animal species that have sustained Indigenous peoples over thousands of years of participating in the natural world” (p. 5). These food systems are sustained through active participation by people; indeed, the demonstrated use of a food or land system has been required for a number of court cases pertaining to First Nations land rights (Morrison, 2011).

Despite the Indigenous origins of food sovereignty through La Via Campesina, the potential of IFS to address food insecurity and create cultural continuity has not been addressed in the literature (Rudolph \& McLachlan, 2013). In Canada, the IFS movement was popularized through the People's Food Policy Program, whose Indigenous Circle proposed four important characteristics of IFS: to return to the agreements of the treaties and reform and redistribute land; to include the Indigenous concepts of harmony with nature in resource-based policy; to address the socioeconomic determinants that are negatively affecting Indigenous health; and to rebuild the relationships between Indigenous peoples and other stakeholders (PFPP, 2010). Morrison (2011) expanded on these characteristics, explaining four principles of IFS: there is a sacred responsibility to the land that must be prioritized over colonial law; community needs should be self-determined; active participation in a food system is necessary; and that policy reform is required to guide and support these principles.

Expressions of IFS through community gardens, country foods programs, culture camps and community food harvesting programs have been documented in recent years. Vazquez's (2011) work with the Oneida Nation of Wisconsin, in the United States, promoted food system revitalization. Mundel and Chapman (2010) reported participants in the Urban Aboriginal Community Garden in British Columbia, Canada viewed the project as decolonization because it helped to reduce dependence. IFS research has also been documented by Rudolph and McLachlan (2013) who share the need for politicized sovereignty to achieve Indigenous food sovereignty in a northern Manitoba First Nations community. Kamal and Thompson (2013) have documented an Indigenous food movement in O-Pipon-Na-Piwin Cree Nation, Manitoba. IFS has also been looked at through a community economic development framework as a way to remove some food production from the market and reclaim local access (Thompson et al., 2011). However, none of this documented work points to the earliest practices of IFS; that is, breastfeeding and the consumption of country foods in infants and has generally been overlooked because breastfeeding tends to be considered a health practice rather than part of a food system. 


\section{Breastfeeding}

Breastmilk is an infant's first nourishment. This gift from mother to child provides more than nutritional sustenance. It is considered the ideal food for infants, and the link between breastfeeding and breastmilk and a range of other health issues for children is well known. Research has demonstrated that infants who are breastfed have fewer respiratory tract (Holberg et al., 1991; Wright, Holberg, Martinez, Morgan, \& Taussig, 1989) and ear infections (Duncan, Holberg, Wright, Martinez \& Taussig, 1993), diarrheal based illnesses (Wright, Bauer, Naylor, Sutcliffe, \& Clark, 1998), and asthma (Wright, Holberg, Taussig, \& Martinez, 2001). In remote, rural communities "breast milk is the most secure and economically advantageous" way to feed infants (Willows \& Batal, 2013, p. 41). Breast milk is an important aspect of food security, especially in communities where access to market food is economically prohibitive, and where access to traditional country food is a challenge due to environmental contamination, decline of species, costs of procurement, and availability of hunters in the family (Willows \& Batal, 2013).

The low proportion of breastfed babies in Indigenous communities must be considered within the larger cultural context of the loss of infant feeding traditions (Dodgson \& Struthers, 2003). Long standing policies and programs that have attempted to assimilate First Nations children such as residential schools have had long standing impacts to childrearing practices. The impact of residential schools resulted in a generational disconnect which inhibited the transfer of "traditional child rearing and infant feeding ways" (Dodgson \& Struthers, 2003, p. 55) because former residential school students would have been taught that their traditional ways were inferior to those of the newcomers. In the early 1900s, there was a gradual move away from breastfeeding in favour of bottle feeding worldwide. This related to concerns over insufficient caloric intake for babies, the physical burden of breastfeeding for women (weight loss and fatigue), as well as the influx of women entering the workforce (Ward, 2000). Related to this was the medicalization of childbirth experienced by women during the early part of the 1900s (Dodgson \& Struthers, 2003). Since the 1970s, women from remote, rural communities in Canada have been required to evacuate to hospitals in larger communities or cities. This mandatory policy has shown a cascade of negative social consequences including decreased breastfeeding (Olson \& Couchie, 2013).

The move towards the apparent convenience associated with bottle feeding resulted in a decline in the practice of breastfeeding for several more years until the mid-1900s where a resurgence of breastfeeding occurred. This return to breastfeeding reflected a disillusionment towards the actual conveniences associated with bottle feeding as well as a concern for the over medicalization of feeding babies (Ward, 2000). First Nations women and their babies, however, did not return to breastfeeding at the same rates as other non-First Nations Canadians at this time, and rates continue to remain low. Health Canada reports that overall 87.3 percent of all Canadian mothers breastfeed or tried to breastfeed their last child, and the initiation rates since 2003 remain stable (Health Canada, 2012). Health Canada also surveyed off reserve [Aboriginal] Indigenous women and notes that their breastfeeding initation rates were lower at 77.8 percent 
than non-[Aboriginal] Indigenous mothers. The World Health Organization recommends exclusive breastfeeding for the first 180 days, and complementary feeding starting at the age of six months with continued breastfeeding to the age of two or beyond (WHO, 2009).

In Manitoba First Nations, there has been a steady decline in breastfeeding and recent statistics indicate that from 2002/03 to 2008/2010 there was an 11.1 percent reduction (54 percent to 42.9 percent) in breastfeeding for any length of time (AMC, 2012). This is much lower than the provincial statistics, which indicate that 80 percent of Manitoba women initiated breastfeeding in 2010, although only half were still breastfeeding after the age of six months (Manitoba Health, 2011). The Chief Public Health Officer's Report on the Health of Manitobans also notes that "breastfeeding rates are lower in the north and in low-income areas" (Manitoba Health, 2011, p. 72).

Martens (2012) discusses the importance of knowledge and attitudes around breastfeeding among First Nations adolescents in Manitoba. In Marten’s work on education interventions among youth, First Nations female participants showed a large increase in breastfeeding beliefs and a large decrease in bottle feeding beliefs and a small to medium increase in breastfeeding attitudes. This type of intervention identified the importance of adolescent breastfeeding education which would result in youth who "were more aware of the positive benefits of breastfeeding, less embarrassed about seeing women breastfeed in public, and more willing to encourage and support people in the choice to breastfeed” (Martens, 2012, p. 254). Oneha and Dodgson (2009) discuss the role of social support for Native Hawaiian mothers as being critical in not only initiating breastfeeding, but persisting. They also describe the traditional practice of breastfeeding among Native Hawaiians as a natural expectation, so women and people observing a woman breastfeeding was not unusual. Native Hawaiians also consider spiritual components to breastfeeding such as rituals and prayers to improve lactation, which functioned as a source of reassurance (Oneha \& Dodgson, 2009).

\section{Country foods}

Country food (also called traditional food or cultural food) was the first table food for First Nations infants up until recent years. While these terms are frequently used inter-changeably, the term traditional is to be considered with caution. As Luppens (2009) noted, it is difficult for researchers that are outsiders to ascribe the term "traditional" to the group in question when there is no experience with what that term describes. Moreover, the term is not flexible, in that foods that are store-bought (salmon or berries, for example) are not considered traditional despite their historic use, because they were purchased. Foods that have been used since European contact, such as flour, are also not considered traditional despite the hundreds of years of use. Country food refers mainly to food that is wild-harvested including wild meat (moose meat, deer, bison), fish, birds, sea mammals, berries and other plants (Power, 2008). Compared to market food, country food is "more nutrititious and more nutrient-dense" and is an important part of the diet for many First Nations people (Power, 2008, p. 96). For many remote, rural First Nations 
communities, the reliance on country food has diminished despite soaring costs of store bought food, which is often flown into communities because of the lack of road access. There is an abundance of literature which points to the connection between increased store bought food and the onset of chronic and lifestyle related disease such as type 2 diabetes, obesity and heart related illness (Kuhnlein \& Receveur, 1996). Cultural food goes beyond sustenance and nutrition for many First Nations people:

Traditionally, Aboriginal diets and consumption patterns arose from complex and holistic food systems that provided health benefits beyond nutrition. Culture-a determinant of health, is intricately tied to traditional Aboriginal foods. Not only are traditional food valued from cultural, spiritual and health perspectives, but the activities involved in their acquisition and distribution allow for the practice of cultural values such as sharing and cooperation. (Earle, 2011, p. 3)

The effects of colonization on the relationship to food has been extensive. As Vernon describes, it is imperative to examine this "ruptured relationship" in order to understand "food's power in community building given historical realities that have informed current relationships to food" (Vernon, 2016, p. 138). Willows, Veugelers, Raine, and Kuhle (2011) discuss the link between food, culture and spirituality:

The cultural worldview held by some Aboriginal peoples is that traditional food by its very nature is health promoting and good to eat. For this reason, in addition to nourishing the body, traditional food-as compared to commercial food-has the advantage of nourishing the mind and spirit, being an anchor to culture and personal well-being, and is an essential agent to promote holistic health. (Willows et al., 2011, p. 6)

In a study about the beliefs around weight gain during pregnancy and lactation among Northern Cree women, the role of country food as an agent of health promotion was discussed. Within the Cree context of health miyupimaatisiiun, or "being alive well", the vision of health extends beyond physiological health and encompasses a much more holistic model, including the role of culture and spirituality (Adelson, 2000). Vallianatos et al. (2006) describe the role of traditional food for mother and infants, "these foods connote more than just health. They also symbolize Cree culture or way of life” (p. 12).

The health benefits for infants and children in consuming country foods have been found in parts of northern Canada (Egeland, Pacey, Cao, \& Sobol, 2010). Infant consumption of country food is an important means to avoid market based foods such as iron fortified cereals which are costly in remote, northern communities. Willows and Batal (2013) suggest "wild meats, many of which were, and continue to be, part of traditional Aboriginal child feeding practices are a culturally appropriate alternative” (p. 44). Relatedly, the concerns over insufficient Vitamin D in breastmilk is often a reason women will provide complementary foods such as country food to infants. The Canadian Paediatric Society recommends that all breastfed 
infants who live above the $55^{\text {th }}$ parallel receive Vitamin D supplementation during periods of the year where the sun is lessened (Godel et al., 2007). The concern over diminished Vitamin D is also linked to childhood rickets. The need for Vitamin D supplementation was confirmed in a study by Willows, Dewailly, and Gray-Donald (2000) in James Bay Cree women and infants living above the $53^{\text {rd }}$ parallel in Quebec. While the supplementation of Vitamin D can be a challenge, this can be offset by the promotion of country food. Dodgson and Struthers (2003) noted that soups, soft foods, and jerky were given during the first year when teething. In terms of weaning, it was also noted that Ojibwe babies were not weaned at specific time periods, and instead the mothers took their cue from the infant in deciding when it was time to stop breastfeeding (Dodgson \& Struthers, 2003). Their participants did note that weaning was done if the mother became pregnant, as it was believed that breasfeeding during pregnancy could "hurt the pregnancy” (Dodgson \& Struthers, 2003, p. 58).

\section{Methodology}

Research in First Nations communities generally requires the establishment and maintenance of trusting relationships (Wilson, 2008). A research relationship was already established with the Norway House Cree Nation Health Division and the investigators as a part of the BTT study. Based on the discussions of interim BTT study findings, and some of the conversations between the community researchers and participants on traditional cultural approaches, a further research partnership was created with Norway House Cree Nation Health Division. This topic required a qualitative approach and involved interviews and focus groups. Using a participatory research approach, we engaged in a partnership with the Norway House Cree Nation Health Division who assisted with the development of research questions, as well as participant recrutiment. As well, a local community member was hired who was also one of the community researchers for the BTT study.

During two one week intensive periods, a total of 20 interviews were held, as well as four focus groups. The participants were primarily grandmothers and great grandmothers. Some of whom were former and current primary health care providers in various capacities, both in traditional health as in the biomedical field. Most of the participants practiced or used traditional medicine or "Indian medicine”. Preliminary findings were presented in large and small group formats as well as one-on-one with stakeholders in the community which assisted with analysis. Knowledge transfer continues to take place as we work towards developing community-based intervention programming in the various mother/child/family programs in the community.

Grounded theory (Strauss \& Corbin, 1994) methodology was used in this project because this approach is particularly suitable when little is known about a topic, such as First Nations approaches to infant feeding. Creswell (1998) provides a definition of grounded theory which aligned with our research goals. Grounded theory is described as: 
....an approach that generates or discovers a theory or an abstract analytical schema of a phenomenon that relates to a particular situation. This situation is one in which individuals interact, take actions, or engage in a process in response to a phenomenon. (Creswell, 1998)

Interviews and focus groups were audio recorded, transcribed, and coded using NVIVO qualitative analysis software. Interview and focus group questions primarily focused on child birthing practices, the role of culture and family in supporting infants, oral health practices and infant feeding practices such as breastfeeding, although this paper focusses on only some of these themes. Interviews were primarily conducted in English, although in some cases the participants chose to speak in Cree, in which case an interpreter was used. The community researcher was well known in the community as working in the area of maternal and children's health in various programs, and she was able to solicit participants through her own networks as well as by using the local radio station. A First Nations undergraduate student from the local university also participated in gathering the data, conducting analysis and writing of this paper (LZ). Another First Nations graduate student with expertise in IFS is the second co-author on this paper (TM). This research was approved under the University of Winnipeg Human Ethics Review Board. In addition, Norway House Cree Nation approved this project put forward by the local Health Division.

\section{Findings and discussion}

Healthy infant feeding traditions are embedded in cultural approaches to childrearing. However, dramatic changes in infant feeding were identified by the research participants as being connected to larger shifts away from cultural based childrearing. The findings indicated that the reliance on medical advice around infant feeding, coupled with the drastic changes in maternal diet including the diminished consumption of country foods, and the influence of processed, high sugar, high sodium food, the low prevalence of breastfeeding, as well as an overall lack of education on infant feeding practices have shaped the "culture" of unhealthy infant feeding in the community. These changes in infant feeding practices cannot be understood within a vacuum, and the wider social and cultural context of the community is required. This article focuses specifically on areas related to breastfeeding practices and the introduction of country food as ways in which women supported the health of their infants and promoted IFS from an early age. This article also acknowledges that there are cultural tensions that exist around these topics. The grandmothers who were interviewed describe childrearing practices embedded in traditional cultural norms earlier in time. They also describe the influence of Western-based medical advice, which often contradicted the approaches they used to rear children. The grandmothers also describe what they see today with their own children and grandchildren who are rearing children in ways that in sometimes go against Western medical 
approaches to health as well as traditional approaches. What is important to consider is that cultures are not frozen but fluid. Overall, the larger influence of colonization as described by Vernon (2016) has disrupted the cultural continuity that mitigated negative health outcomes.

\section{Breastfeeding practices}

The participants within our study noted that breastfeeding was a socially acceptable practice when they were having babies. It was not uncommon to see women in the community breastfeeding in public spaces. One of our oldest respondents discussed how she would openly breastfeed:

... at the store if he was hungry I would breastfeed right there. If there was no chair I would ask for one and say 'I want to breastfeed my baby', so they would [provide a chair]. I was not shy because everybody was breastfeeding then.

The participants considered breastfeeding as a natural practice in which they felt encouraged by their family and community. As one participant told us, "It came naturally to breastfeed. My aunt came and talked to me about it while I was pregnant on how it is important to breastfeed.”

Discussion of infant feeding is intimately linked to discussions around birthing practices. Like many women living in remote, rural Canadian communities, the women of Norway House are not allowed to deliver their babies in their home community and are sent to the nearest city hospital in Thompson or Winnipeg. Until the 1970s, women in Norway House were able to birth at the community hospital unless they were assessed to have a high-risk pregnancy or potential delivery, at which time they were taken to a tertiary center for care. Due to the age of most of the respondents, they primarily gave birth in Norway House prior to the mandatory evacuation policy. They delivered their babies surrounded by their spouses, children, family and friends. For new mothers, having support from family was an essential component of healthy infant feeding practices. Some participants felt supported and well advised on childrearing when they were having children. One respondent said, "I think breastfeeding came from my grandmother. My grandmother always instilled in us that breastfeeding was the best way”. Another woman (humorously) describes how she shares the importance of breastfeeding with young women, "they (Elders) always wanted the mothers to breastfeed their kids. That's why god gave you those things (breasts)! Those are not play things! That's how they explained it to you; for your babies not for your husbands!”

Mentorship from Elders including grandparents, aunties, and mothers was important for not only practicing healthy childrearing methods, but also for social support. During the time when the participants were having babies and raising children, women were living with extended families which enabled a supportive environment for childrearing. One participant describes: 
...in those days we lived with families. We didn't have a home. We had to live with my mother in law. She did a lot of teaching on how to look after my children when they get sick. ...they were my great teachers.

Another respondent discussed the role of extended family:

That was the roles of my aunties. They taught me how to take care of my babies, how to feed them, how to not to put too much stuff so they won't get stomach aches. They used to teach them how to give them water but while breastfeeding. They told me I didn't need to give them anything but my breast.

This support was described by another participant, “At the time I was having babies I had support from older ladies that have knowledge of breastfeeding”.

Support and mentorship is essential for women who are initiating breastfeeding, especially if it is for the first time. Adequate support also means that women will feel more encouraged to continue breastfeeding. Oneha and Dodgson (2009) describe this cultural and emotional support as being comprised of "presence, encouragement, expertise and experience" (p. 85) from both family members as well as those in the health field. This support and mentorship also provided an opportunity for women to continue breastfeeding. One participant stated, "I learned a lot from them: the way they did it, they changed her for me; cleaned her up and they did everything for me. This helped me to continue breastfeeding." Participants also discussed trying to support their grandchildren who are now having children themselves and some of the generational gaps. As one respondent described:

...back then you were taught to listen to what the elders tell you, you did it. You didn't question. You just did it because that was our upbringing. You don't talk back; you do the thing you're told to do and that's what I did.

\section{Introduction of country food}

The research participants discussed at length the role of country food (such as fish, moose meat, rabbit) as being an important part of the early diets of infants for several reasons including health and prevention of illness, and an appreciation for this type of food as adults. When the research participants were having babies, there was limited store bought baby food available other than infant cereal. One respondent described, "there was no baby food like what they have today."

Respondents also discussed the advice given by their doctors as conflicting with the traditional advice they were given by mothers, grandmothers and aunties.

...they give you information on how to feed your children...not feeding them until babies are three to six months. There was no 
such thing as that. We started feeding our children at least when they were 1 month or 2 months.

One respondent discussed at length what she saw being practiced by other mothers in the community.

I know they used to start feeding their kids early. I didn't feed my kids solid food until they were 3 months. When they turned one is when I gave them baby food or wild soup as they got older. Duck soup, goose soup, rabbit soup stuff like that. Babies did like that.

Dodgson and Struthers (2003) found similar responses where country food was given at the same time as the commencement of teething. Another respondent discussed the differences she sees in current infant feeding practices: "From my experience with my granddaughter and daughters, they follow what the health nurses tell them about not feeding them until 6 months.” This recommendation would have been in line with the recommendations from the World Health Organization (2009) on the introduction of complementary foods at six months; however, the grandmother's suggestion of earlier introduction of country foods would fall below the WHO recommendations.

Respondents discussed the various ways in which they were taught to feed infants with country food. One respondent described how she ignored the recommendation of the doctors to feed her babies at a later age and instead followed the advice of her grandmother.

We started feeding them with the food we would eat. We would chew it up for them and give it to them. There was no such thing as mashing it... we do it ourselves with our mouth. We chew it first then putting it in baby's mouth and teaching them how to eat.

This insistency in going against the recommendations of physicians is interesting and likely challenging for many health care providers to understand without an adequate cultural understanding and the larger impacts of cultural continuity for First Nations health. A study by Van Esterik (2002) shows the role of the mother as primary decision maker around appropriate times for introducing table food, particularly given the environmental and cultural dimensions of childrearing; this is also linked to the interplay between cultural norms and physician recommendations. Another respondent indicated other methods for feeding country food, "I started to give them squished table food: fish, rabbits, potatoes. They liked it. Babies will eat everything. They liked fish soup and broth. I used to put it in their bottle.” One woman discussed how she alternated breastfeeding and table food feeding.

...between breastfeeding I gave them water. I used to give them wild food with a spoon. I gave them fish water. It is when you boil white fish and make broth. They were about 4 months when I started giving them wild food.

This practice is likely linked to the issue of iron and Vitamin D and iron deficiency in northern communities described by Willows and Batal (2013). 
The importance of introducing nutrient dense food such as country food (Power, 2008) is even more important, considering that women who were unable to breastfeed had little alternatives for providing adequate childhood nutrition. As the Canadian Pediatric Standards have indicated in 2007 (well after any of our participants had babies), for infants residing north of the $55^{\text {th }}$ parallel, Vitamin D supplementation should be provided. Another factor is that many of the research participants had children close in age, and they would have likely weaned their babies off the breast while pregnant because of cultural beliefs around breastfeeding harming the pregnancy (Dodgson \& Struthers, 2003). The early introduction of country food to the infant's diet was considered to be intimately linked to improved health and the prevention of illness. A participant stated, "It was good they were healthy. The white fish, we would make a stew out of that and feed it to our kids. They were healthy. They hardly ever got sick. Nowadays I see children get sick so easily”. All the women within the study were able to identify that an infant feeding regiment based on breastmilk as well as the early introduction of country food, resulted in the best infant health outcomes.

The links between cognitive development and the consumption of country food was described by many respondents. As one participant indicated,

I was told that your kids would be bright if you eat fish. You need it (fish) to be smart, you have to eat fish. I've seen studies today that show that mothers that eat fish have kids with high IQs.

Children that eat chips have lower IQs.

Information on infant feeding was part of the knowledge transmission passed down through generations. Like traditional medicine, country food is often called "medicine” in First Nations culture because of the more holistic and spiritual perspectives on health. This is similar to what Adelson (2000) describes, "the nutritional value of meat is connected to the significance of the animal powers or spirits. The larger and more powerful animals have a greater nutritional value and are thus viewed as stronger foods” (Adelson, 2000, p. 80).

\section{Reframing Indigenous food sovereignty to include first foods}

As a means of producing and consuming foods, breastfeeding and the introduction of country foods as first foods are examples of Indigenous food sovereignty, and part of a living culture. These practices help to revitalize traditional knowledge which includes traditional childrearing practices. Respondents in this project, while they were describing infant feeding practices in the context of early childhood caries, were also talking about the ability to make choices that were considered culturally appropriate and effective methods of childrearing that in many cases are not in line with national and international recommendations on infant feeding. Breastmilk as a first food has demonstrably provided a range of health protecting factors for childhood development and health. Country foods, as described by participants, was considered an essential supplement to breastfeeding for early infants and another first foods. 
These practices enabled mothers and families to adequately support their children's immediate needs, but were also a part of a larger local food system, thereby reducing reliance on a market-based food system. The women in this study stated that they belonged to larger support networks among extended family and community members that helped to guide them with breastfeeding practices as well as the acquisition of country foods. These are all tenets of Indigenous food sovereignty.

Cultural continuity has a protective influence on physical and mental health (Chandler and Lalonde, 1998) for First Nations communities. Other scholars have suggested that cultural continuity is a social determinant of health (Loppie-Reading \& Wein, 2009). Cultural continuity is equally important when considering food systems. Changes in traditional food knowledge and family dynamics have resulted in the need for education and support around traditional food practices including first foods. As Morrison (2011) notes, "the Indigenous food sovereignty approach provides a model for social learning and thereby promotes the application of traditional knowledge, values, wisdom, and practices in the present day context” (p. 100). The transmission of food-related knowledge, including infant feeding practices, serves to promote cultural continuity.

The social element of these practices should not be overlooked. According to Fouts, Hewlett, and Lamb (2012), "Breastfeeding involves much more than feeding; it entails intimate social interactions that reflect cultural ideas and practices about social relationships, childcare, and child development.” (p. 123). IFS is a concept to be considered, described, and supported, but caution must be used in ascribing this term to a people or a place. In the end, a community must define its own food practices and priorities, but IFS accounts must consider breastfeeding and country foods as first foods in the first steps towards the path to IFS and put cultural continuity at the heart.

Acknowledgements: This research was funded through the New Investigator Award for Aboriginal scholars through the Network Environment for Aboriginal Health Research (NEAHR) through the Canadian Institute of Health Research. We would like to acknowledge the support of the Manitoba NEAHR program in assisting with the logistics of this project. Funding was also provided through Northern Scientific Research Program. We would also like to acknowledge the Norway House Cree Nation Health Division for their ongoing gracious hosting and support of this project. The women who shared with us their knowledge on childrearing, breastfeeding and oral health reminded us of the importance of women as culture carriers.

\section{References}

Adelson, N. (2000). 'Being Alive Well': Health and the Politics of Cree Well-being (Vol. 16). University of Toronto Press. 
AMC (Assembly of Manitoba Chiefs), 2012. First Nations Regional Health Survey (RHS) Phase 2 (2008-2010): Manitoba Regional Report. Winnipeg: Assembly of Manitoba Chiefs, Revised November 2012.

Chandler, M.J. \& Lalonde, C. (1998). Cultural continuity as a hedge against suicide in Canada's First Nations. Transcultural Psychiatry, 35(2), 191-219.

Chandler, M.J. \& Dunlop, W.L. (2015). Cultural Wounds Demand Cultural Medicines (pp. 7890). Toronto, Ontario: Canadian Scholars’ Press Inc.

Creswell, J. W. (2013). Research design: Qualitative, Quantitative, and Mixed Methods Approaches. Sage publications. Dodgson, J., \& Struthers, R. (2003). Traditional breastfeeding practices of the Ojibwe of Northern Minnesota. Health Care for Women International, 24(1), 49-61.

Duncan, B., Ey, J., Holberg, C.J., Wright, A.L., Martinez, F.D., \& Taussig, L.M. (1993). Exclusive breast-feeding for at least 4 months protects against otitis media. Pediatrics, 91(5), 867-872.

Earle, L. (2011). Traditional Aboriginal Diets and Health. National Collaborating Centre on Aboriginal Health. Retrieved from https://www.ccnsa-nccah.ca/docs/emerging/FSTraditionalDietsHealth-Earle-EN.pdf

Egeland, G.M., Pacey, A., Cao, Z., \& Sobol, I. (2010). Food insecurity among Inuit preschoolers: Nunavut Inuit child health survey, 2007-2008. Canadian Medical Association Journal, 182(3), 243-248.

Fouts, H.N., Hewlett, B.S., \& Lamb, M.E. (2012). A biocultural approach to breastfeeding interactions in Central Africa. American Anthropologist, 114(1), 123-136.

Godel, J. C., Irvine, J., Onyett, H., Saylor, K., Schroter, H., \& Young, M. (2007). Vitamin D supplementation: recommendations for Canadian mothers and infants. Paediatr Child Health, 12(7), 583-9.

Kamal, A.G. \& Thompson, S. (2013, September). Recipe for decolonization and resurgence: Story of O-Pipon-Na-Piwin Cree Nation's indigenous food sovereignty movement. In Conference Paper (Vol. 46).

Kuhnlein, H.V. \& Receveur, O. (1996). Dietary change and traditional food systems of indigenous peoples. Annual Review of Nutrition, 16(1), 417-442.

Health Canada. Breastfeeding Initiation in Canada: Key Statistics and Graphics (2009-2010). 2012. Retrieved from: www.hc-sc.gc.ca/fnan/surveill/nutrition/commun/prenatal/initiation-eng.php (Accessed December 5, 2012). 
Health Canada. (2012). Breastfeeding Initiation in Canada: Key Statistics and Graphics (20092010). Available at: http://www.hc-sc.gc.ca/fnan/surveill/nutrition/commun/prenatal/initiation-eng.php (Accessed December 5, 2012).

Holberg, C.J., Wright, A.L., Martinez, F.D., Ray, C.G., Taussing, L.M., Lebowitz, M.D., \& Group Health Medical Associates. (1991). Risk factors for respiratory syncytial virusassociated lower respiratory illnesses in the first year of life. American Journal of Epidemiology, 133(11), 1135-1151.

Lardeau, M.P., Healey, G., \& Ford, J. (2011). The use of Photovoice to document and characterize the food security of users of community food programs in Iqaluit, Nunavut. Rural and Remote Health, 11(2), 1680.

Lawrence, H.P., Cidro, J., Isaac-Mann, S., Peressini, S., Maar, M., Schroth, R. J., ... \& Jamieson, L. (2016). Racism and oral health outcomes among pregnant Canadian Aboriginal women. Journal of Health Care for the Poor and Underserved, 27(1), 178-206.

Lawrence, H.P., Binguis, D., Douglas, J., McKeown, L., Switzer, B., Figueiredo, R., \& Laporte, A. (2008). A 2-year community-randomized controlled trial of fluoride varnish to prevent early childhood caries in Aboriginal children. Community Dentistry and Oral Epidemiology, 36(6), 503-516.

Lawrence, H., Romanetz, M., Rutherford, L., Cappel, L., Binguis, D., \& Rogers, J. B. (2004). Oral health of aboriginal preschool children in northern Ontario. Probe, 38(4), 172-190.

Loppie Reading, C. \& Wein, F. (2009). Health Inequalities and Social Determinants of Aboriginal Peoples’ Health. Prince George, BC: National Collaborating Centre for Aboriginal Health.

Luppens, L. (2009). Today Indian Food: Perspectives of Aboriginal Peoples on the Foods in their Contemporary Diets. Unpublished Master's thesis, Queen’s University, Kingston, ON. https://qspace.library.queensu.ca/handle/1974/5703?show=full

Manitoba Health (2011). Priorities for Prevention: Everyone, Every Place, Every Day. Chief Provincial Public Health Officer's Report on the Health Status of Manitobans 2010. Presented by Joel Kettner, Chief Provincial Public Health Officer. As retrieved from http://www.gov.mb.ca/health/cppho/pfp.pdf. (Revised November 1, 2011).

Martens, P.J. (2012). What do Kramer’s Baby-Friendly Hospital Initiative PROBIT studies tell us? A review of a decade of research. Journal of Human Lactation, 28(3), 335-342.

Martens, T. (2015). Good News in Food: Understanding the Value and Promise of Indigenous Food Sovereignty in Western Canada. Unpublished Master's thesis, University of Manitoba, Winnipeg, MB. 
Morrison, D. (2008). Working Group on Indigenous Food Sovereignty Final Activity Report. BC Food Systems Network.

Morrison, D. (2011). Indigenous food sovereignty: A model for social learning. In H. Wittman, A. Desmarais, \& N. Wiebe (eds.), Food sovereignty in Canada: Creating Just and Sustainable Food Systems, Fernwood Publishing, Halifax, NS, 97-113.

Mundel, E. \& Chapman, G. E. (2010). A decolonizing approach to health promotion in Canada: The case of the Urban Aboriginal Community Kitchen Garden Project. Health Promotion International, 25(2), 166-173.

Olson, R. \& Couchie, C. (2013). Returning birth: The politics of midwifery implementation on First Nations reserves in Canada. Midwifery, 29(8), 981-987.

Oneha, M.F.M. \& Dodgson, J.E. (2009). Community influences on breastfeeding described by Native Hawaiian mothers. Pimatisiwin-A Journal of Aboriginal and Indigenous Community Health, 7(1), 75-97.

PFPP (People’s Food Policy Project). 2010. First principles protocol for building cross-cultural relationships [online]. Retrieved from foodsecurecanada.org/sites/foodsecurecanada.org/fi les/First_Principles_July_2010.pdf.

Power, E.M. (2008). Conceptualizing food security for Aboriginal people in Canada. Canadian Journal of Public Health, 99, 95-97.

Reading, J. (2015). Confronting the growing crisis of cardiovascular disease and heart health among Aboriginal peoples in Canada. Canadian Journal of Cardiology, 31(9), 10771080.

Reeds, J., Mansuri, S., Mamakeesick, M., Harris, S.B., Zinman, B., Gittelsohn, J., \& Hanley, A. (2016). Dietary patterns and type 2 diabetes mellitus in a First Nations community. Canadian Journal of Diabetes, 40(4), 304-310.

Rudolph, K.R. \& McLachlan, S.M. (2013). Seeking Indigenous food sovereignty: Origins of and responses to the food crisis in northern Manitoba, Canada. Local Environment, 18(9), 1079-1098.

Skinner, K. (2006). Disparity in a prosperous land: getting enough affordable, good quality food is difficult in remote Aboriginal communities. Alternatives Journal, 32(3), 29-30.

Strauss, A. \& Corbin, J. (1994). Grounded theory methodology. Handbook of Qualitative Research, 17, 273-85. 
Thompson, S., Lozeznik, V., Gulrukh, A., Ballard, M., Islam, D., Beardy, B., \& Wong, K. (2011). Is community economic development putting healthy food on the table? Food sovereignty in northern Manitoba's Aboriginal communities. Journal of Aboriginal Economic Development, 7, 33-45.

Thompson, S., Kamal, A.G., Alam, M.A., \& Wiebe, J. (2012). Community development to feed the family in northern Manitoba communities: Evaluating food activities based on their food sovereignty, food security, and sustainable livelihood outcomes. Canadian Journal of Nonprofit and Social Economy Research, 3(2), 43-66.

Turner, N.J. \& Turner, K.L. (2008). "Where our women used to get the food”: Cumulative effects and loss of ethnobotanical knowledge and practice; case study from coastal British Columbia. Botany, 86(2), 103-115.

Tobe, S.W., Maar, M., Roy, M.A., \& Warburton, D.E. (2015). Preventing cardiovascular and renal disease in Canada's Aboriginal populations. Canadian Journal of Cardiology, 31(9), 1124-1129.

Vallianatos, H., Brennand, E. A., Raine, K., Stephen, Q., Petawabano, B., Dannenbaum, D., \& Willows, N. D. (2006). Beliefs and practices of First Nation women about weight gain during pregnancy and lactation: Implications for women's health. Canadian Journal of Nursing Research, 38(1), 102-119.

Van Esterik, P. (2002). Contemporary trends in infant feeding research. Annual Review of Anthropology, 31(1), 257-278.

Vazquez, J.M. (2011). The role of indigenous knowledge and innovation in creating food sovereignty in the Oneida Nation of Wisconsin. Unpublished Master's thesis, Iowa State University, Ames, IA. https://www.mobt3ath.com/uplode/book/book-14094.pdf

Vernon, R.V. (2016). A Native perspective: Food is more than consumption. Journal of Agriculture, Food Systems, and Community Development, 5(4), 137-142.

Ward, D., Wiley, M., \& Smithing, R. (2000). Policies, issues and trends affecting practice. In Meredith \& Horan (Eds.), Adult Primary Care. W.B.Saunders, Philadelphia, PA.

Willows, N., Dewailly, E., \& Gray-Donald, K. (2000). Anemia and iron status in Inuit infants from northern Quebec. Canadian Journal of Public Health, 91(6), 407-10.

Willows, N. (2005). Determinants of healthy eating in Aboriginal peoples in Canada: The current state of knowledge and research gaps. Canadian Journal of Public Health, 93, S32-S36. 
Willows, N., Veugelers, P., Raine, K., \& Kuhle, S. (2011). Associations between household food insecurity and health outcomes in the Aboriginal population (excluding reserves). Health Reports, 22, 15-20.

Willows, N. \& Batal, M. (2013). Nutritional concerns of Aboriginal infants and children in remote and northern Canadian communities: Problems and therapies. In Nutrition in Infancy, Humana Press, 39-49.

Wilson, S. (2008). Research is Ceremony: Indigenous Research Methods. Fernwood Publishing: Halifax, NS.

WHO (World Health Organization). (2009). Infant and Young Child Feeding: Model Chapter for Textbooks for Medical Students and Allied Health Professionals. Retrieved from: http://apps.who.int/iris/bitstream/10665/44117/1/9789241597494_eng.pdf?ua=1\&ua=1

Wright, A.L., Holberg, C.J., Martinez, F.D., Morgan, W.J., \& Taussig, L.M. (1989). Breastfeeding and lower respiratory tract illness in the first year of life. British Medical Association Journal, 299(6705), 946-949.

Wright, A.L., Bauer, M., Naylor, A., Sutcliffe, E., \& Clark, L. (1998). Increasing breastfeeding rates to reduce infant illness at the community level. Pediatrics, 101(5), 837-844.

Wright, A.L., Holberg, C.J., Taussig, L.M., \& Martinez, F.D. (2001). Factors influencing the relation of infant feeding to asthma and recurrent wheeze in childhood. Thorax, 56(3), 192-197. 